\title{
Supplementary information for "Evaluating an exponential respiration model to alternative models for soil respiration components in a Canadian wildfire chronosequence (FireResp, v1.0),
}

John Zobitz ${ }^{1, *}$, Heidi Aaltonen ${ }^{2, *}$, Xuan Zhou ${ }^{3, *}$, Frank Beninger ${ }^{3, *}$, Jukka Pumpanen $^{2, *}$, and Kajar Köster, *

${ }^{1}$ Augsburg University, Minneapolis, Minnesota, United States

${ }^{2}$ Department of Environmental and Biological Sciences, University of Eastern Finland, Kuopio, Finland

${ }^{3}$ Department of Environmental and Biological Sciences, University of Eastern Finland, Joensuu, Finland

${ }^{4}$ Department of Forest Sciences, University of Helsinki, Helsinki, Finland

${ }^{*}$ These authors contributed equally to this work.

Correspondence: Kajar Köster (kajar.koster@helsinki.fi)

Competing interests. The authors declare no competing interests. 


Qield approach; $5 \mathrm{~cm}$ depth

\begin{tabular}{lllllllll}
\hline \multicolumn{10}{c}{ Field approach; $10 \mathrm{~cm}$ depth } \\
\hline$Q_{10, M}$ & $Q_{10, R}$ & $k_{R}$ & $k_{S}$ & $k_{M}$ & $k_{A}$ & $\mu$ & $\varepsilon$ \\
\hline
\end{tabular}

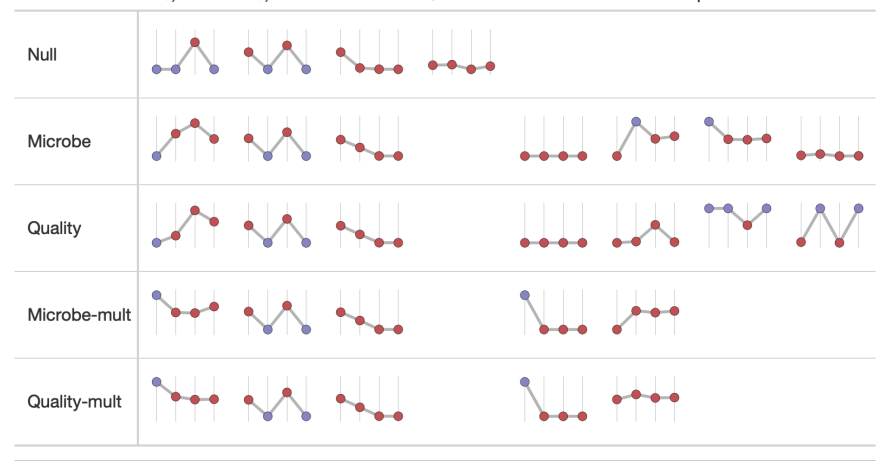

\begin{tabular}{lllllllll}
\hline \multicolumn{10}{c}{ Field approach; All depths } \\
\hline$Q_{10, M}$ & $Q_{10, R}$ & $k_{R}$ & $k_{S}$ & $k_{M}$ & $k_{A}$ & $\mu$ & $\varepsilon$ \\
\hline
\end{tabular}

Quality-mult

Figure S1. Median values of parameter estimates for different models using the Field parameter estimation approach. The horizontal axis on each sparkline plot is arranged by the year since the burn sites in the chronosequence (2012, 1990, 1968, or Control). In each row the vertical axis scale is the same. Edge hitting parameters are denoted with the blue coloring. 


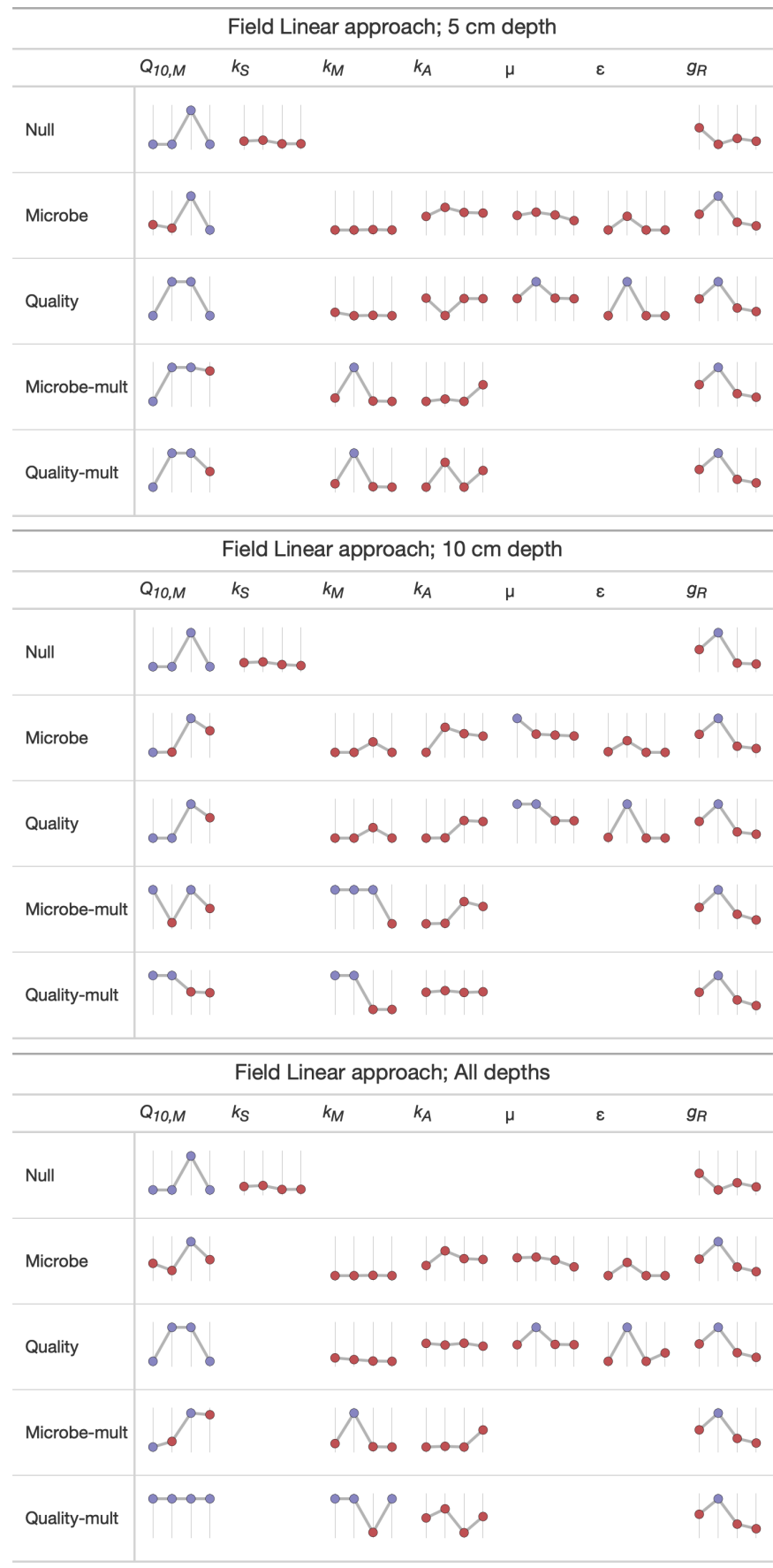

Figure S2. Median values of parameter estimates for different models using the Field Linear parameter estimation approach. The horizontal axis on each sparkline plot is arranged by the year since the burn sites in the chronosequence $(2012,1990,1968$, or Control). In each row the vertical axis scale is the same. Edge hitting parameters are denoted with the blue coloring. 


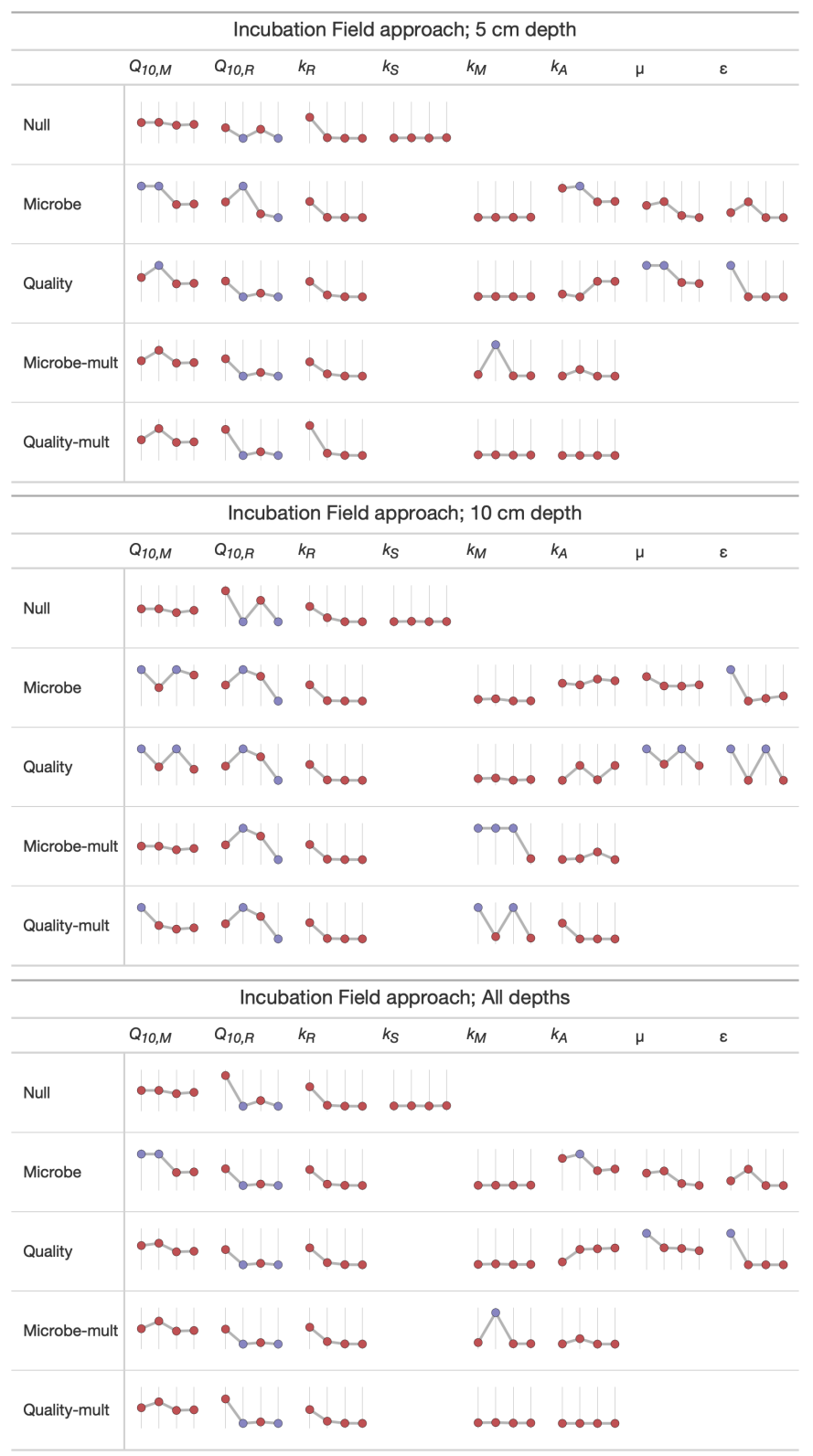

Figure S3. Median values of parameter estimates for different models using the Incubation Field parameter estimation approach. The horizontal axis on each sparkline plot is arranged by the year since the burn sites in the chronosequence (2012, 1990, 1968, or Control). In each row the vertical axis scale is the same. Edge hitting parameters are denoted with the blue coloring. 


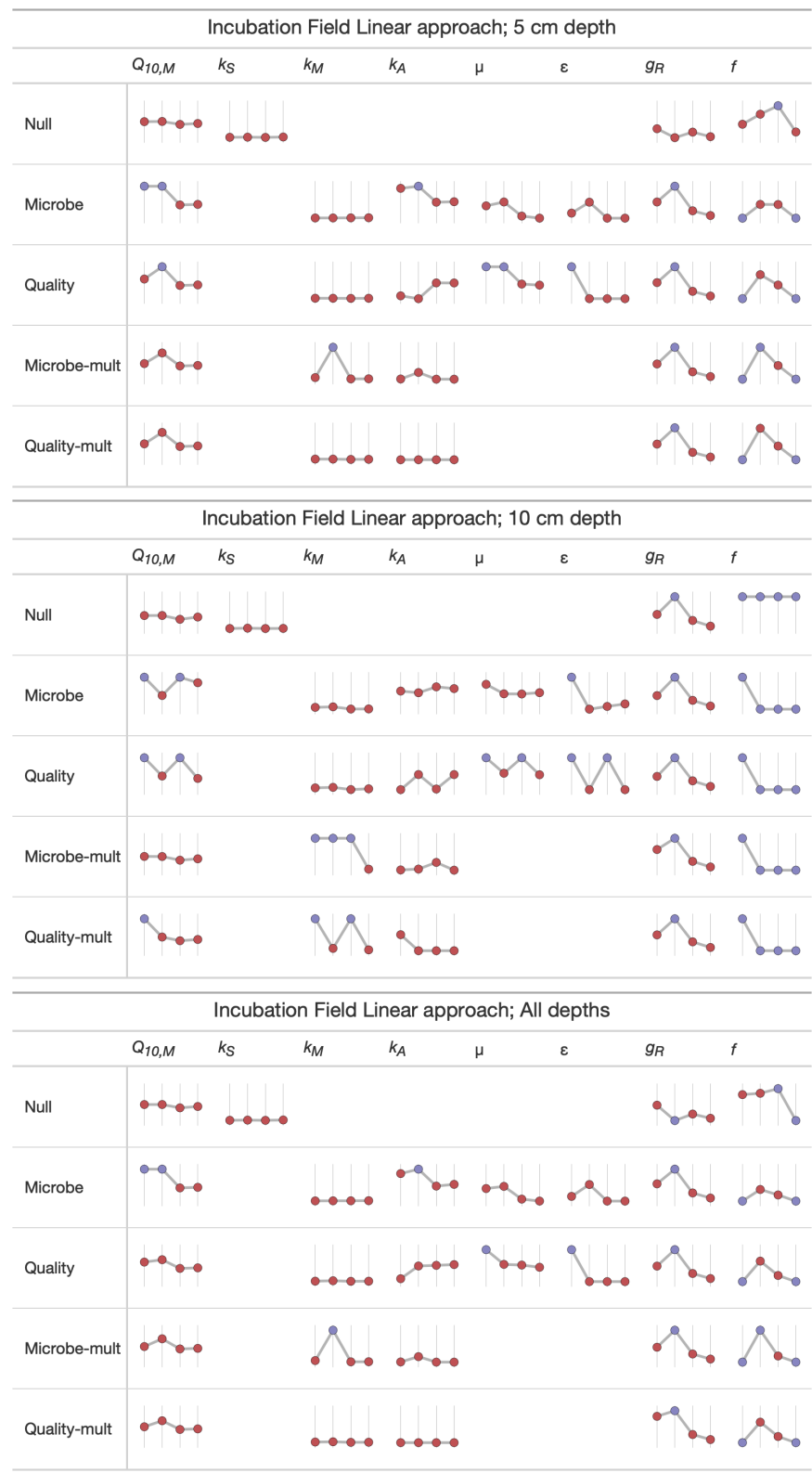

Figure S4. Median values of parameter estimates for different models using the Incubation Field Linear parameter estimation approach. The horizontal axis on each sparkline plot is arranged by the year since the burn sites in the chronosequence (2012, 1990, 1968, or Control). In each row the vertical axis scale is the same. Edge hitting parameters are denoted with the blue coloring. 